\title{
Yogic Sleep and Walking Protocol Induced Improvement in Sleep and Wellbeing in Post-menopausal Subject: A Longitudinal Case Study During COVID Lockdown
}

\author{
Kamalesh K. Gulia ${ }^{1}$ (D) Sapna Erat Sreedharan ${ }^{2}$
}

Received: 3 August 2021 / Revised: 10 October 2021 / Accepted: 29 October 2021 / Published online: 12 November 2021

(c) The Author(s), under exclusive licence to Springer Nature Singapore Pte Ltd. 2021

\begin{abstract}
Purpose Post-menopausal life is fairly long period of life that is marked by poor health and sleep. Fatigue amidst extraordinary pandemic stress had taken a toll on the sleep quality and overall wellbeing. Yogic sleep can be instrumental in relaxing the brain and help in achieving self-control of mind and body in the post-menopausal life. This can be a non-pharmacological intervention to improve the wellbeing of women.

Methods Effect of 24 weeks of yoga-nidra practice and exercise module was tested in a post-menopausal subject after taking baseline of 4 weeks on parameters like sleep latency, total sleep time, mood on waking and during day, BMI, and activity rhythm of body using $24 \mathrm{~h}$ actigraphy and sleep diary.

Results After administering the dual protocol, there was remarkable elevation in mood both on waking up and entire day from 5th week onwards. Mood shifted toward a happier state. Latency to sleep decreased after 4 weeks, while total sleep time improved only after 16 weeks of dual management strategy. The BMI was also reduced to 28.4 from initial value of 30.3. Morning awakening patterns did not change, but it was not accompanied by pain or headache.

Conclusion The results indicated the therapeutic potential of yoga-nidra and exercise package in this actigraphy-based longitudinal pilot study. Yoga-nidra can be easily practiced at home, and thus, it is a promising non-pharmacological strategy for aging population in improving their wellbeing.
\end{abstract}

Keywords Yoga-nidra $\cdot$ Post-menopausal $\cdot$ Wellbeing $\cdot$ Mood $\cdot$ Sleep

\section{Introduction}

Women spent nearly one-third of their life in climacteric. Deterioration in mood and a decreased feeling of wellbeing are common observations in post-menopausal women facing age-related physiological changes [1]. A change in the quantity and quality of sleep is often suggested to cause

Kamalesh K. Gulia

kkguliak@hotmail.com

Sapna Erat Sreedharan

sapnaerat@sctimst.ac.in

1 Division of Sleep Research, Department of Applied Biology, Biomedical Technology Wing, Sree Chitra Tirunal Institute for Medical Sciences and Technology, Trivandrum, Kerala 695012, India

2 Department of Neurology, Comprehensive Centre for Sleep Disorders, Sree Chitra Tirunal Institute for Medical Sciences and Technology, Trivandrum, Kerala 695011, India these changes [2, 3]. Moreover, extended lockdowns due to the new corona virus disease (COVID-19) has brought social isolation, reduced mobility, and unprecedented stress. Stress-induced reduction in physical activity and a sedentary lifestyle further aggravate sleep and mental health [4]. Additional discomforts due to accompanying headaches and pain, which usually occur during the post-menopausal stage, only worsen the general health conditions. In this direction, measures for attaining Good Health and Wellbeing, one of the Sustainable Developmental Goal (SDG-3) by the United Nations is timely and significant. It is emphasized that this pandemic period was associated with a decline in sleep quality and a disruption of circadian rhythms even in population unaffected by COVID-19 [5-7]. Though, in postmenopausal women, there are studies on strategies like walking program, body mind exercise, and yoga [8-11], there are no studies on the beneficial effects of yoga-nidra (yogic sleep) in this age group during this pandemic period. Yoga is increasingly recognized as a universally accepted science to 
achieve physical and mental wellbeing, therapeutic potential of yoga-nidra with exercise module was tested on the health in this longitudinal pilot study.

\section{Methods}

Informed consent was taken from 56 year old female subject who completed menopause 5 years back. No history of hypertension, diabetes, or any neuropsychiatric disease was found. Subject experienced frequent early morning awakenings, fatigue, body pain, stress, and low mood. She underwent a baseline actigraphy (Somnowatch plus ${ }^{\circledR}$ from Somnomedics) for 4 weeks starting from 13 August 2020 for long-term objective measurement of sleep patterns. Actigraphy was continued during 24 weeks of practice of yoga-nidra and walking exercise protocol with effect from 10 Sept 2020. Yoga-nidra intervention developed by Swami Satyananda Saraswati from Bihar School of Yoga (Munger, India) was employed every morning using instructions from audio $\mathrm{CD}$ obtained from the Ashram [12]. Yoga-nidra was practiced for $27 \mathrm{~min}$ on working days, and for $48 \mathrm{~min}$ on weekends and holiday (audio CD session) in morning from 5 am to $9 \mathrm{am}$. Basically yoga-nidra technique involves systematic observation of several steps while lying down in shavasana (in corpse pause with eyes closed) beginning with preparation involving internalization, taking resolve (sankalpa), rotation of consciousness, breathing awareness, manifestations of opposites, image visualization, resolve and externalization, or return to full awareness at the end. A conscious systematic control of brain makes this technique more special and powerful to attain enhanced relaxed state by integrating body and mind. A sleep diary (devised by the National Sleep Foundation) was maintained by the subject throughout the study period. Sleep latency and duration were calculated along with pattern (biphasic or monophasic sleep). Questions pertained to wellbeing including (1) mood on waking was noted and scored (Refreshed-1, somewhat-2, or fatigued-3); (2) mood during day noted in evening (bad -4 , very bad-3, happy -2 , or very happy-1); (3) pain (yes -1 or no-0) were noted every day. A refreshed state of mind on waking is a reflection of recuperative sleep. Fatigueness due to poor sleep can also affect the day time mood in addition to contribution from other stressful events during day (external factors). Weight and height were also fed in actigraph Domino Light software to calculate changes in body mass index (BMI). Brisk walking was carried out in evening for 25-30 min mostly indoors in lobby and occasionally outdoors. Actiwatch was worn by the subject throughout $(24 \mathrm{~h}$ X 28 weeks); it was taken out only at the time of washing or data transfer. For preliminary comparison, pre-interventions data-scores of 4 week parameters including mood (on waking and daytime) for wellbeing, BMI, sleep latency, and duration were compared with post-interventions scores (every 4 week) using $t$ test. The significance level was set at $p<0.05$.

\section{Results}

Before the yoga-nidra intervention, during COVID lockdown period, the activity levels were far lesser as subject was not motivated for any indoor or outdoor exercises (Fig. 1A). However, after administering the dual protocol, significant changes were observed in mood from 5th week onwards in both morning and entire day as the feeling of fatigue on waking up gradually reduced with increased instances of waking up refreshed (Table 1). Daytime mood shifted toward happier state. Latency to sleep (assessed subjectively based on scores) decreased after 4 weeks, while total sleep time improved only after 16 weeks of dual management strategy (Table 1). The BMI was also reduced to 28.4 from initial value of 30.3. With implementation of yoga-nidra technique, subject experienced dramatic reduction in the headache and neck pain associated with early morning awakenings from 5 th week without altering the patterns of early morning awakenings, i.e., biphasic pattern of sleep did not change (Fig. 1). Studied parameters appeared to stabilize after 20th week of practice.

\section{Discussion}

Marked improvement in the emotional state of subject after practice of yoga-nidra clearly indicated that relaxing the brain and strengthening self-determination component would have helped in getting over the fatigue mounted by sedentary life amidst stressful COVID lockdowns. This definitely helped in resuming and regularizing the walking exercise. Yoga-nidra practice is shown to reduce anxiety as it is considered much higher state than several other relaxation techniques wherein mere relaxation of physical body (muscles) may be achieved. Therefore, this technique has a potential in achieving deep relaxation of mind and reducing the stress levels. It is used as a therapeutic tool for management of various ailments like depression, menstrual disorders, insomnia, and for attaining overall mental health [12-16]. Regular practice of yoga-nidra along with walking exercise improved sleep latency and feeling of satisfied sleep. It is well known that walking exercise alone could help in burning calories, and thus, it helped in improving BMI. However, it remains a challenge to involve people in activities in postmenopausal stage due to lethargy and reduced self-drive. Yoga-nidra reduces anxiety and brings self-control. Walking would aid in balancing the circadian rhythm of the body and in association with yoga-nidra associated relaxed mind 
Fig. 1 Actigraph-based 24 activity profile before and 20-24 weeks of yoga-nidra and walking intervention. Activity plot showing pre-intervention baseline (BL) of 4 weeks starting from 13 August to 9 September 2020 (A) and effects of dual intervention at 20 weeks from 13 January to 9 February 2021 (B) and 24 weeks, 10 February to 8 March 2021 (C) from 12:00 afternoon to 12:00 the next day. Red broken lines are depicting early morning awakenings, while blue broken lines are depicting the gross time in sleep
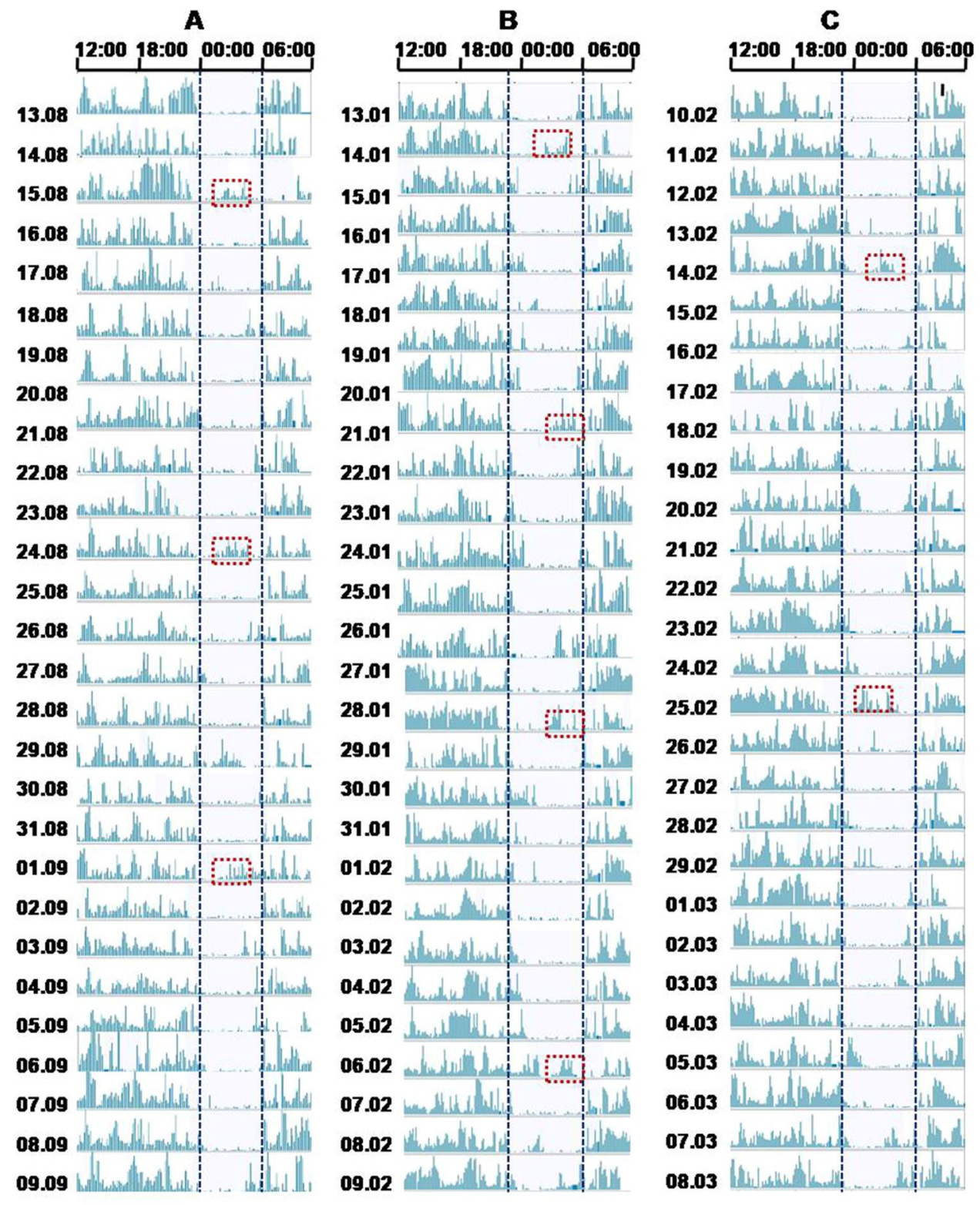

Pre-Intervention BL

20 wk Post-lntervention

Table 1 Averaged 4-weekly scores of mood on waking, mood during the day, BMI; latency to sleep, and actual time in sleep (hr:min)

\begin{tabular}{|c|c|c|c|c|c|}
\hline S. no. & Mood on waking (scores) & Mood during day (scores) & BMI (scores) & Latency to sleep (scores) & TST (hr:min) \\
\hline Pre & $2.44 \pm 0.51$ & $1.96 \pm 0.34$ & $30.3 \pm 0.2$ & $2.07 \pm 0.55$ & $6.09 \pm 1.38$ \\
\hline Post 1 & $2.29 \pm 0.59$ & $1.87 \pm 0.43$ & $29.5 \pm 0.3$ & $1.52 \pm 0.51 * *$ & $6.21 \pm 1.33$ \\
\hline Post 2 & $1.83 \pm 0.54 * *$ & $1.59 \pm 0.50 *$ & $29.2 \pm 0.0 * *$ & $1.45 \pm 0.51^{* *}$ & $6.30 \pm 1.39$ \\
\hline Post 3 & $1.59 \pm 0.68 * *$ & $1.41 \pm 0.73 * *$ & $29.3 \pm 0.4^{*}$ & $1.28 \pm 0.45^{* *}$ & $6.39 \pm 1.59$ \\
\hline Post 4 & $1.38 \pm 0.56^{* *}$ & $1.07 \pm 0.26^{* *}$ & $29.3 \pm 0.2 * *$ & $1.45 \pm 0.69 * *$ & $6.46 \pm 1.44$ \\
\hline Post 5 & $1.39 \pm 0.58^{* *}$ & $1.13 \pm 0.46^{* *}$ & $29.3 \pm 0.2 *$ & $1.21 \pm 0.49^{* *}$ & $7.13 \pm 1.22 * *$ \\
\hline Post 6 & $1.38 \pm 0.56^{* *}$ & $1.14 \pm 0.35^{* *}$ & $28.7 \pm 0.2 * *$ & $1.24 \pm 0.58 * *$ & $6.53 \pm 1.34 *$ \\
\hline
\end{tabular}

All values are presented as mean \pm SD for pre (pre-intervention values of 4 weeks) and post (average of every 4-weekly data after practice of yoga-nidra and walking exercise). Mood scores on waking up (Fatigued-3, Somewhat-2, Refreshed-1); Mood during the day (V Bad-4, Bad-3, Happy-2, V Happy-1); BMI; latency to sleep (easy, i.e., less than $15 \mathrm{~min}$, score 1; after some time, i.e., 15-45 min, score 2; and difficult, i.e., more than 45 min with score of 3), total sleep time (TST) in hr:min. $* * *$ depicts comparison with baseline $(*<0.05, * *<0.001)$. Pre refers to 4 weeks of baseline recording, Post denotes six bins of 4-weekly data on administering the dual protocol 
would help in uplifting the overall mood on waking and during the day [8]. Recuperative sleep is essential for mood stability especially this fag end of life. Good quality sleep and uplifted mood during the day also helped in conducting the routine work more effectively.

It is noted that morning awakenings were not affected by this dual strategy as it is possible that common age-related organic changes may have set in this subject. However, interestingly, alleviation of the adverse events associated with morning awakenings (pain and headaches) indicated therapeutic potential of yoga-nidra. In yoga-nidra practice, one accomplishes sleep like state consciously but actually without getting into the unconscious sleep or full awakening. The preliminary results obtained from this case report clearly indicate the therapeutic potential of yoga-nidra and exercise package based on this actigraphy-based longitudinal pilot study.

As per the ancient Indian literature, along with nutrition, sleep and exercise are two pillars of good health and sleep can be improved using several strategies according to traditional School of Indian Medicine [17]. Yoga is a broad science comprising of processes of restraining chitta (state of mind) from any fluctuation. Recent meta-analysis also showed that yoga improves the sleep quality in women [18]. Yoga-nidra particularly engages Pratyahara, which is fifth element of Ashtanga yoga as per Patanjali Yoga that involves disassociation of consciousness/senses from the outside environment. As post-menopausal age is marked by sleep disorders and related health issues, there is need to pay attention to sleep health and bringing in appropriate management measures in place [19]. It must be remembered that nearly half the world cannot afford gold standard therapies like cognitive behavior therapy due to gross lack of such expertise, facilities, and expenses involved. In this light, yoga-nidra is a promising non-pharmacological technique for post-menopausal women when they are coping with several physiological unavoidable changes affecting their health and wellbeing. Moreover, longer life expectancy in women compared to men also highlights an additional requirement of a viable management strategy to alleviate their sufferings and pain amidst poor sleep [20].

\section{Conclusion}

It is emphasized that yoga-nidra is a special technique in which profound relaxation similar to naturally occurring deep sleep is consciously attained to derive complete physical, mental, and emotional relaxation. It can be easily done at home setup making it a non-pharmacological technique with tremendous potential in regulation of mind and body. Under these circumstances of the mounting stress of COVID pandemic and otherwise current sedentary lifestyle, there is dire need of revisiting a yoga strategy in a big randomized controlled trial, so that can be utilized by larger set of aging population to improve their wellbeing amidst a simple home setup.

Author Contributions KKG contributed to conceptualization, planning and execution of the study, and manuscript preparation. SES contributed to conceptualization and manuscript preparation.

Funding None.

Availability of Data and Materials Actigraphy records are available if asked for.

Code Availability (Software Application or Custom Code) Not applicable.

\section{Declarations}

Conflict of Interest None.

Ethics Approval Informed consent of subject was taken (not required in case study).

Consent to Participate (Include Appropriate Statements) Not applicable.

Consent for Publication (Include Appropriate Statements) Yes.

\section{References}

1. Monteleone P, Mascagni G, Giannini A, et al. Symptoms of menopause - global prevalence, physiology and implications. Nat Rev Endocrinol. 2018;14:199-215.

2. Moreno-Frías C, Figueroa-Vega N, Malacara JM. Relationship of sleep alterations with perimenopausal and postmenopausal symptoms. Menopause. 2014;21:1017-22.

3. Li DX, Romans S, De Souza MJ, et al. Actigraphic and selfreported sleep quality in women: associations with ovarian hormones and mood. Sleep Med. 2015;16:1217-24.

4. Creasy SA, Crane TE, Garcia DO, et al. Higher amounts of sedentary time are associated with short sleep duration and poor sleep quality in postmenopausal women. Sleep. 2019;42:zsz093.

5. Blume C, Schmidt MH, Cajochen C. Effects of the COVID-19 lockdown on human sleep and rest-activity rhythms. Curr Biol. 2020;30:R795-7.

6. Morin CM, Carrier J, Bastien C, et al. Canadian sleep and circadian network. Sleep and circadian rhythm in response to the COVID-19 pandemic. Can J Public Health. 2020;111:1-4.

7. Zhao X, Lan M, Li H, et al. Perceived stress and sleep quality among the non-diseased general public in china during the 2019 coronavirus disease: a moderated mediation model. Sleep Med. 2020;:S1389-9457(20):30224.

8. Tadayon M, Abedi P, Farshadbakht F. Impact of pedometer-based walking on menopausal women's sleep quality: a randomized controlled trial. Climacteric. 2016;19:364-8. 
9. Buchanan DT, Landis CA, Hohensee C, et al. Effects of yoga and aerobic exercise on actigraphic sleep parameters in menopausal women with hot flashes. J Clin Sleep Med. 2017;13:11-8.

10. Duman M, Taşhan ST. The effect of sleep hygiene education and relaxation exercises on insomnia among postmenopausal women: a randomized clinical trial. Int J Nurs Pract. 2018;24:e12650.

11. Sydora BC, Turner C, Malley A, et al. Can walking exercise programs improve health for women in menopause transition and postmenopausal? Findings from a scoping review. Menopause. 2020;27:952-63.

12. Saraswati SS. Yoga Publishing Trust. Munder, Bihar. 2001

13. Rani K, Tiwari S, Singh U, Agrawal G, Ghildiyal A, Srivastava N. Impact of Yoga Nidra on psycho-biological general wellbeing in patients with menstrual irregularities: a randomized controlled trial. Int J Yoga. 2011;4:20-5.

14. Sang-Dol K. Psychological effects of yoga nidra in women with menstrual disorders: a systematic review of randomized controlled trials. Complement Ther Clin Pract. 2017;28:4-8.

15. Datta K, Tripathi M, Mallick HN. Yoga nidra: an innovative approach for management of chronic insomnia-a case report. Sleep Sci Pract. 2017;1(1):1-11.

16. Ozdemir A, Saritas S. Effect of yoga nidra on the self-esteem and body image of burn patients. Complement Ther Clin Pract. 2019;35:86-91.
17. Gulia KK, Radhakrishnan A, Kumar VM. Approach to sleep disorders in the traditional school of indian medicine: alternative medicine II. In: Sleep disorders medicine: basic science, technical considerations and clinical aspects. (4th Edition) Chapter 57, 2017, pp 1221-1232 (ISBN 978-1-4939-6578-6).

18. Wang W, Chen K, Pan Y, et al. The effect of yoga on sleep quality and insomnia in women with sleep problems: a systematic review and meta-analysis. BMC Psychiatry. 2020;20:195.

19. Caretto M, Giannini A, Simoncini T. An integrated approach to diagnosing and managing sleep disorders in menopausal women. Maturitas. 2019;128:1-3.

20. Gulia KK, Kumar VM. Sleep disorders in the elderly: a growing challenge. Psychogeriatrics. 2018;18:155-65.

Publisher's Note Springer Nature remains neutral with regard to jurisdictional claims in published maps and institutional affiliations. 